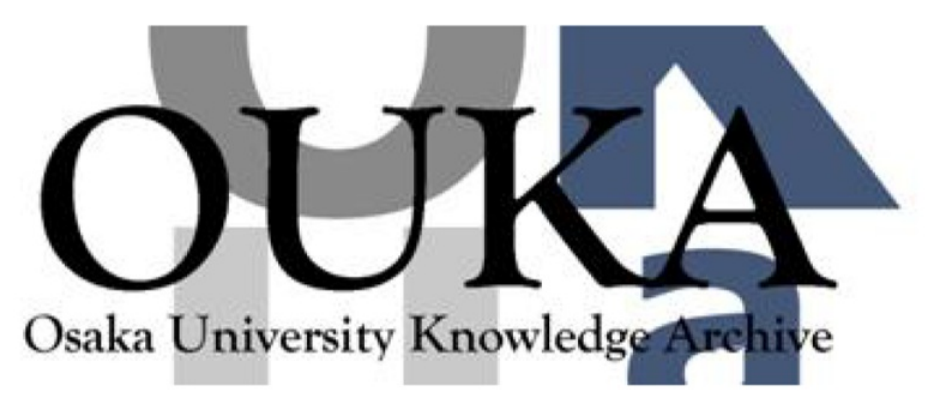

\begin{tabular}{|c|l|}
\hline Title & $\begin{array}{l}\text { Threshold improvement in uniformly lying helix } \\
\text { cholesteric liquid crystal laser using } \\
\text { auxiliary } \pi \text {-conjugated polymer active layer }\end{array}$ \\
\hline Author(s) & $\begin{array}{l}\text { Yoshida, Hiroyuki; Shiozaki, Yusuke; Inoue, Yo } \\
\text { et al. }\end{array}$ \\
\hline Citation & $\begin{array}{l}\text { Journal of Applied Physics. 113(20) p. 203105- } \\
\text { p. 203105 }\end{array}$ \\
\hline Issue Date & $2013-05-23$ \\
\hline oaire:version & VoR \\
\hline URL & https://hdl. handle. net/11094/75672 \\
\hline rights & \\
\hline Note & \\
\hline
\end{tabular}

Osaka University Knowledge Archive : OUKA

https://ir. Library. osaka-u. ac. jp/

Osaka University 


\section{Threshold improvement in uniformly lying helix cholesteric liquid crystal laser using auxiliary $\pi$-conjugated polymer active layer}

Cite as: J. Appl. Phys. 113, 203105 (2013); https://doi.org/10.1063/1.4807402

Submitted: 01 March 2013 . Accepted: 06 May 2013 . Published Online: 23 May 2013

Hiroyuki Yoshida, Yusuke Shiozaki, Yo Inoue, Masaya Takahashi, Yasuhiro Ogawa, Akihiko Fujii, and Masanori Ozaki
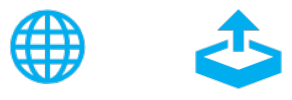

\section{ARTICLES YOU MAY BE INTERESTED IN}

A stable and switchable uniform lying helix structure in cholesteric liquid crystals

Applied Physics Letters 99, 041108 (2011); https://doi.org/10.1063/1.3615293

Position sensitive, continuous wavelength tunable laser based on photopolymerizable cholesteric liquid crystals with an in-plane helix alignment

Applied Physics Letters 94, 093306 (2009); https://doi.org/10.1063/1.3089846

Electrically controllable laser based on cholesteric liquid crystal with negative dielectric anisotropy

Applied Physics Letters 88, 061122 (2006); https://doi.org/10.1063/1.2168259

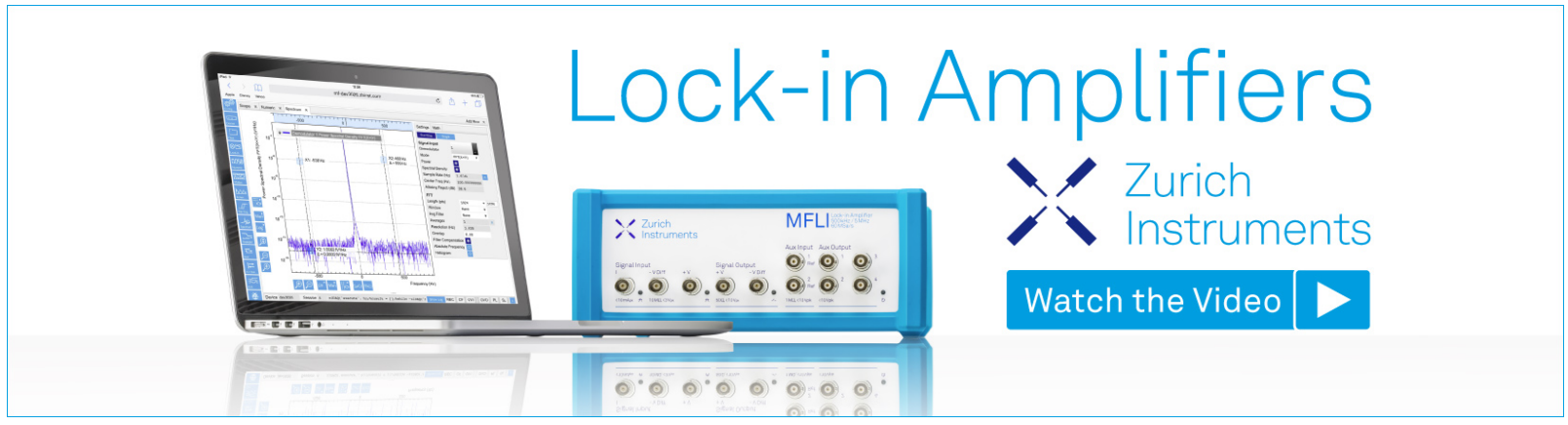




\title{
Threshold improvement in uniformly lying helix cholesteric liquid crystal laser using auxiliary $\pi$-conjugated polymer active layer
}

\author{
Hiroyuki Yoshida, ${ }^{\text {a) }}$ Yusuke Shiozaki, Yo Inoue, Masaya Takahashi, Yasuhiro Ogawa, \\ Akihiko Fujii, and Masanori Ozaki \\ Division of Electrical, Electronic and Information Engineering, Graduate School of Engineering, \\ Osaka University, 2-1 Yamada-oka, Suita, Osaka 565-0871, Japan
}

(Received 1 March 2013; accepted 6 May 2013; published online 23 May 2013)

\begin{abstract}
We propose a device structure to lower the lasing threshold of a uniformly lying helix cholesteric liquid crystal (ChLC) laser. We place a $\pi$-conjugated polymer active layer beneath the ChLC layer to provide auxiliary gain, and demonstrate an improvement in the lasing threshold by a factor of 2.3. We also perform finite difference time domain calculations coupled with rate equations for a four-level system, and clarify the effect of the additional active layer on both the photonic density of states and the inversion population density. Although the addition of an extra layer lowers the photonic density of states, the gain provided by the auxiliary layer is sufficient to overcome the losses and decrease the lasing threshold. Our concept is useful for obtaining high-performance ChLC lasers. (C) 2013 AIP Publishing LLC. [http://dx.doi.org/10.1063/1.4807402]
\end{abstract}

\section{INTRODUCTION}

Cholesteric liquid crystals (ChLCs) are known to selforganize into helical structures with periodicities of a few hundred nanometers and therefore possess a reflection band between $n_{o} p$ and $n_{e} p$, where $n_{o}, n_{e}$, and $p$ are the ordinary refractive index, extraordinary refractive index, and helical pitch, respectively. Owing to the enhancement of the density of states (DOS) at the reflection band edge, laser action can be realized by doping an appropriate gain medium (usually laser dyes) into ChLCs. In addition, the stop band can be easily controlled by external stimuli such as temperature, electric field, light, and elasticity. Consequently, ChLCs have attracted interest as compact, low-cost, and tunable coherent light sources. ${ }^{1-3}$

Recent studies on ChLC lasers have focused on developing new materials and/or device structures to improve the performance, i.e., the threshold and the efficiency, of the device. One of the most popular approaches to lowering the threshold of ChLC laser devices is to increase the photonic DOS at the lasing mode. This has been achieved, for example, by introducing a structural defect in the perfect helical lattice, ${ }^{4-6}$ or by confining ChLCs between two reflecting surfaces, such as a pair of dielectric multilayers. ${ }^{7,8}$ On the other hand, while the simplest approach to increasing the photonic DOS is to increase the cavity length of the helical periodic structure, ${ }^{9}$ implementing this concept in a conventional ChLC laser with planar alignment (with the helical axis oriented perpendicular to the substrates) has not been so successful, because exponential decay of the excitation light owing to absorption leads to a non-uniformity in the inversion population density, and sets a maximum cavity length above which the threshold starts to increase. ${ }^{10}$

We have previously demonstrated that lowering of the threshold by increasing the cavity length becomes possible if the helical axis is oriented in the cell-plane direction.

a)e-mail: yoshida@eei.eng.osaka-u.ac.jp
Compared with the thickness of the cell, which is usually a few micrometers thick, aligning the helix in the cell-plane direction allows the cavity to be much longer $(\sim \mathrm{mm})$, leading to a dramatic enhancement of the DOS. Using this so-called uniformly lying helix (ULH) structure, we demonstrated reduction in the lasing threshold by more than a factor of 10 compared with planar ChLC lasers. ${ }^{11,12}$

Another important parameter related to the threshold characteristics of a laser is the gain. In a dye-doped ChLC laser, the net gain attainable is not very high, even with the use of a laser dye, because the device is small and the amount of dye that can be doped in the ChLC is limited by the onset of quenching. However, while extensive studies have been performed to enhance the photonic DOS, studies devoted to enhancing the gain of the laser are limited since it is dependent on the development of new materials with high fluorescent properties. ${ }^{13}$ In this study, we show that the gain of the system can be increased by inserting an auxiliary gain layer in a ULH ChLC laser between the ChLC layer and the substrate, and that it is effective in further reducing the lasing threshold. We experimentally demonstrate reduction in the lasing threshold by a factor of 2.3 by employing a fluorescent $\pi$-conjugated polymer active layer. We also clarify the effect of the auxiliary active layer on the photonic DOS and the total gain achieved, by analyzing the system by the finite difference time domain (FDTD) method including auxiliary differential equations (ADEs) describing stimulated emission from a four-level gain medium. ${ }^{14-17}$

\section{EXPERIMENTAL PROCEDURE}

Two samples were prepared in this study: a ULH ChLC laser with the auxiliary active layer and a reference sample without the active layer. The ChLC material was prepared by doping a right-handed chiral dopant (Merck, R-811) and a laser dye 2-[2-4(dimethylamino)phenyl]ethenyl- 6-methyl4H-pyran-4-ylidene propanedinitrile (Exciton, DCM), at concentrations of 33 and 1.0 wt. \%, respectively, in a nematic 


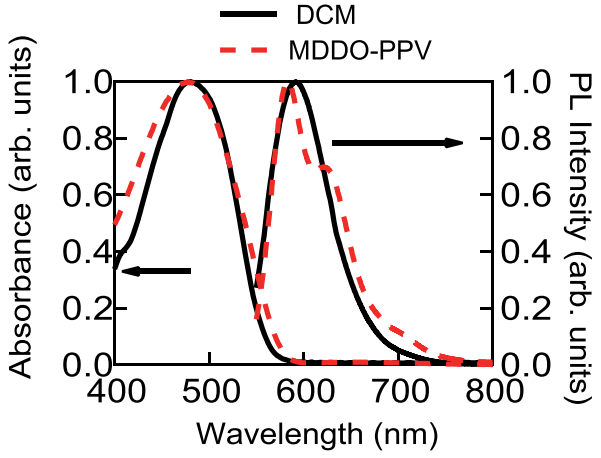

FIG. 1. Absorbance and PL spectra of DCM and MDDO-PPV.

LC host (Merck, E44). This material has been investigated before and is known to have the long edge of the selective reflection band at $\sim 600 \mathrm{~nm} .{ }^{12,18}$ A $\pi$-conjugated polymer, poly(2-methoxy-5-dodecyloxy-p-phenylenevinylene) (MDDO-PPV) was synthesized in house ${ }^{19,20}$ and used as the auxiliary gain medium. Figure 1 shows absorption and photoluminescence spectra of the two materials. The materials have approximately the same absorption and fluorescence peaks, indicating that both materials can contribute equally to laser action.

The laser device was fabricated by assembling a sandwich cell using two glass substrates, and infiltrating the ChLC in the cell gap. Of the two substrates, one had interdigitated electrodes made from $\mathrm{Cr} / \mathrm{Au}(20 \mathrm{~nm} / 80 \mathrm{~nm})$, with an electrode-gap of $100 \mu \mathrm{m}$. MDDO-PPV was spin-coated onto the substrate without the electrodes to form a film approximately $0.5 \mu \mathrm{m}$ thick. The substrates were coated with a thin polyimide layer to induce homeotropic alignment (JSR, JALS-2021-R2) and assembled into a sandwich cell with a gap of $\sim 1 \mu \mathrm{m}$. The ChLC was infiltrated into the cell above the clearing point $\left(75^{\circ} \mathrm{C}\right)$ and was cooled to $27.5^{\circ} \mathrm{C}$ at a rate of $1{ }^{\circ} \mathrm{C} / \mathrm{min}$, while applying a square-wave electric field with an amplitude and frequency of $1.0 \mathrm{~V} / \mathrm{m}$ and $1 \mathrm{kHz}$, respectively, between the electrodes. ${ }^{12}$ The reference sample was prepared by the same procedure, except that the MDDOPPV layer was not coated on the substrate. ULH alignment

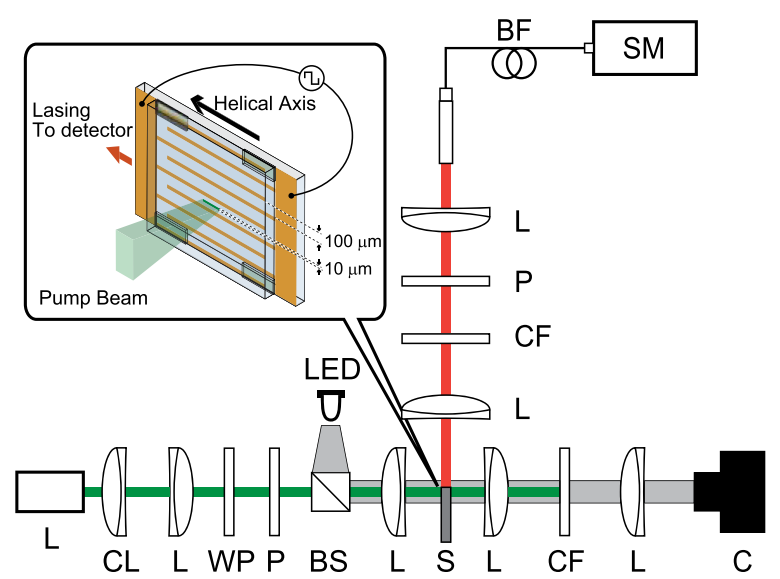

FIG. 2. Optical setup of the laser experiment. L: Pump Laser; CL: Cylindrical Lens; L: Lens; WP: Wave Plate; P: Polarizer; BS: Beam Splitter; S: Sample; CF: Color Filter; BF: Bundle Fiber; SM: Spectrometer with CCD detector; C: USB Camera. of the ChLC between the electrodes was confirmed by polarized optical microscopy.

The lasing characteristics of the two samples were evaluated by optically pumping the sample with the second harmonic $(\lambda=532 \mathrm{~nm})$ of a mode-locked Nd:YAG laser (Ekspla, PL2201) with pulse width and repetition rate of $100 \mathrm{ps}$ and $100 \mathrm{~Hz}$, respectively. The excitation beam was focused using relay optics to excite a rectangular area approximately $100 \mu \mathrm{m}$ long and $10 \mu \mathrm{m}$ wide, with the long axis parallel to the electrodes (along the helical axis). The emitted light was measured with a spectroscope (Horiba, TRIAX320) and CCD detector (Horiba, Symphony) in the direction parallel to the substrates, i.e., along the helix axis. The experimental setup is schematically shown in Fig. 2.

\section{SIMULATION}

To describe the lasing phenomenon occurring in the ChLC, we coupled Maxwell's equations with rate equations of the electron population in a four-level gain medium, and performed numerical calculations using the FDTD algorithm. ${ }^{21,22}$ The time-dependent electromagnetic field propagating in the $x-z$ plane obeys the following Maxwell's equations:

$$
\begin{gathered}
\Delta \times \boldsymbol{E}(x, z, t)=-\mu_{0} \frac{\partial \boldsymbol{H}(x, z, t)}{\partial t}, \\
\Delta \times \boldsymbol{H}(x, z, t)=\varepsilon_{0} \tilde{\varepsilon}(z) \frac{\partial \boldsymbol{E}(x, z, t)}{\partial t}+\frac{\partial \boldsymbol{P}(x, z, t)}{\partial t},
\end{gathered}
$$

where $\varepsilon_{o}$ and $\mu_{0}$ are the dielectric permittivity and the magnetic permeability in vacuum, respectively. $\boldsymbol{P}$ is the polarization density, which provides gain in the system. We describe $\boldsymbol{P}$ using the Lorentz oscillator model

$$
\frac{d^{2} \boldsymbol{P}}{d t^{2}}+\Delta \omega_{a} \frac{d \boldsymbol{P}}{d t}+\omega_{a}^{2} \boldsymbol{P}=-\frac{\gamma_{r}}{\gamma_{c}} \frac{e^{2}}{m} \Delta N \boldsymbol{E},
$$

where $\Delta \omega_{a}=1 / \tau_{21}+2 / T_{2}$ is the full width at halfmaximum (FWHM) linewidth of luminescence, $\tau_{i j}(i, j=0$, $1,2,3)$ is the lifetime of transition from states $i$ to $j, T_{2}$ is the mean time between dephasing events, $\omega_{a}=2 \pi c / \lambda_{a}$ is the central frequency of emission, $\Delta N(x, z, t)$ is the difference between electron numbers at levels 2 and $1, \gamma_{r}=1 / \tau_{21}$, and $\gamma_{c}=e^{2} / m \cdot \omega_{a} /\left(6 \pi \varepsilon_{0} c^{3}\right)$ is the classical rate related to radiation based on electron transition, where $e$ is the electron charge, $m$ is the electron mass, and $c$ is the speed of light in vacuum.

The electron densities at each energy level $\left(N_{0}, N_{1}, N_{2}\right.$, and $N_{3}$ ) follow the rate equations:

$$
\begin{gathered}
\frac{d N_{3}}{d t}=P_{r} N_{0}-\frac{N_{3}}{\tau_{32}}, \\
\frac{d N_{2}}{d t}=\frac{N_{3}}{\tau_{32}}+\frac{1}{\hbar \omega_{a}} \boldsymbol{E} \cdot \frac{\partial \boldsymbol{P}}{\partial t}-\frac{N_{2}}{\tau_{21}}, \\
\frac{d N_{1}}{d t}=\frac{N_{2}}{\tau_{21}}-\frac{1}{\hbar \omega_{a}} \boldsymbol{E} \cdot \frac{\partial \boldsymbol{P}}{\partial t}-\frac{N_{1}}{\tau_{10}},
\end{gathered}
$$




$$
\frac{d N_{0}}{d t}=-P_{r} N_{0}+\frac{N_{1}}{\tau_{10}},
$$

where $P_{r}$ is the pumping rate of the electrons in the ground state $N_{0}$ to the highest excited state $N_{3}$. The total electron density $N=N_{0}+N_{1}+N_{2}+N_{3}$ is constant and depends on the type of active medium.

The behavior of the system upon exciting the system is simulated by assuming an arbitrary pump rate $P_{r 0}$ and investigating how $\boldsymbol{E}$ and $\boldsymbol{H}$ evolve with time. However, in the experiment, pumping is not uniform, but decays exponentially because of light absorption. To account for this effect, we provided an exponentially decaying pump rate in the direction of the impinging pump beam according to the equation:

$$
P_{r}(x)=P_{r 0} \times e^{(-\alpha x)},
$$

where $\alpha$ is the absorption coefficient of the gain medium. The emitted beam, on the other hand, was assumed to propagate without loss in the ChLC.

The two types of ChLC laser were analyzed using the two-dimensional models illustrated in Fig. 3. The dimensions of the device were the same as those described in the experimental section except that the length of the cavity was set to $30 \mu \mathrm{m}$ to shorten the calculation time, and the polyimide alignment layer was not included because it was extremely thin $(\sim 10 \mathrm{~nm})$ and smaller than the spatial grid size used for calculation. The pump beam was assumed to impinge on the sample from the $-x$ direction (gradually decaying in the $+x$ direction), and light propagating in the $+z$ direction was monitored to investigate the lasing phenomenon. The parameters used to describe each material are summarized in Tables I and II. The total electron density in MDDO-PPV was estimated from the value of DCM using the fact that the electron density is directly proportional to the absorption coefficient (assuming that the two media have the same

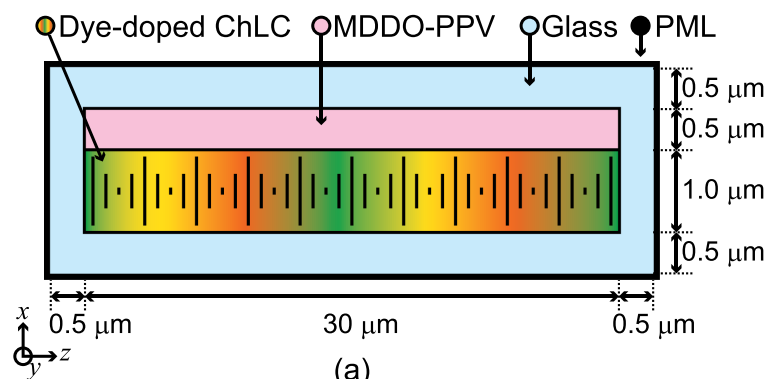

(a)

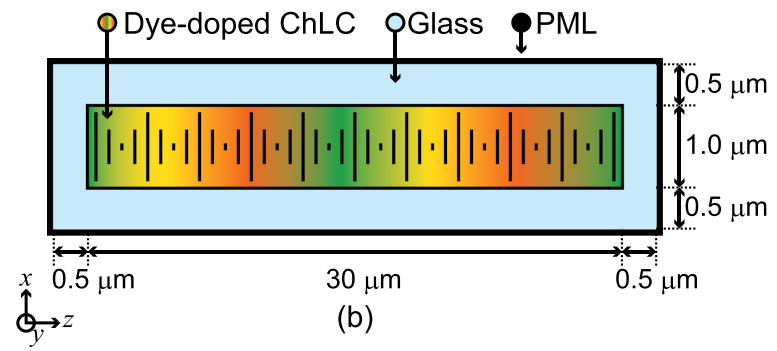

FIG. 3. ChLC laser models in FDTD calculation (a) with and (b) without MDDO-PPV active layer. The pump beam is assumed to impinge on the sample from the $-x$ direction.
TABLE I. Material parameters of ChLC, MDDO-PPV layer, and substrate. Value of $N$ for the ChLC was taken from Ref. 9. Values of $\alpha$ were obtained experimentally.

\begin{tabular}{lrccc}
\hline \hline Material & $n$ & $p(\mathrm{~nm})$ & $N\left(\mathrm{~cm}^{-3}\right)$ & $\alpha\left(\mathrm{cm}^{-1}\right)$ \\
\hline ChLC & $n_{o}=1.50 n_{e}=1.70$ & 353 & $2.4 \times 10^{19}$ & $1.1 \times 10^{3}$ \\
MDDO-PPV & 1.69 & $\ldots$ & $1.3 \times 10^{21}$ & $6.0 \times 10^{4}$ \\
Substrate & 1.50 & $\ldots$ & 0 & 0 \\
\hline \hline
\end{tabular}

TABLE II. Parameters used to solve the rate equations.

\begin{tabular}{lcccc}
\hline \hline$\lambda_{a}(\mathrm{~nm})$ & $T_{2}(\mathrm{~s})$ & $\tau_{32}(\mathrm{~s})$ & $\tau_{21}(\mathrm{~s})$ & $\tau_{10}(\mathrm{~s})$ \\
\hline 600 & $7.63 \times 10^{-15}$ & $1 \times 10^{-13}$ & $1 \times 10^{-9}$ & $1 \times 10^{-11}$ \\
\hline \hline
\end{tabular}

absorption profile). ${ }^{23}$ Because of the similarity of the fluorescence properties, the same values were used for the relaxation times of MDDO-PPV and DCM, choosing values similar to those available in the literature. ${ }^{10,24-27}$ Perfectly matched layer absorbing boundaries were placed around the device to eliminate interference effects, ${ }^{28}$ and the space and time steps, $\Delta x$ and $\Delta t$, were chosen to be $20 \mathrm{~nm}$ and $4.71 \times 10^{-17} \mathrm{~s}$, respectively.

\section{RESULTS AND DISCUSSION}

Figure 4 shows the experimental and calculated lasing spectra of the ChLC lasers with and without the auxiliary MDDO-PPV active layer. Regardless of the presence of MDDO-PPV, a sharp emission peak was obtained near $600 \mathrm{~nm}$, in agreement with theoretical expectations, $\lambda_{\text {lasing }}=n_{e} p$. There was a slight offset in the lasing wavelength between the experiment and simulation, which is believed to have resulted from fluctuations in the pitch and cell thickness. Also, the difference in the lasing wavelength between the two devices in the simulation is likely attributed to the difference in the effective refractive index of the two waveguides. The circular polarization of the emitted beam shown in the inset of the figure, however,

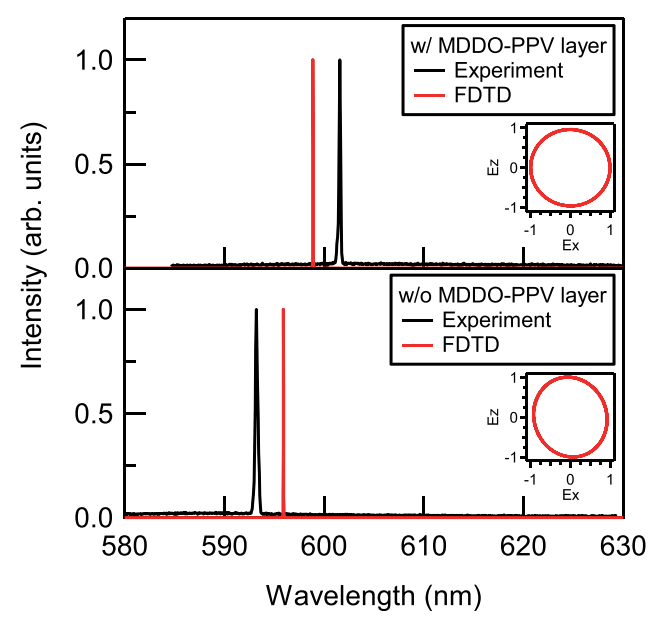

FIG. 4. Emission spectra of ChLC laser (a) with and (b) without MDDOPPV active layer, obtained by experiment and FDTD calculations. Inset shows the polarization of the emitted beam. 


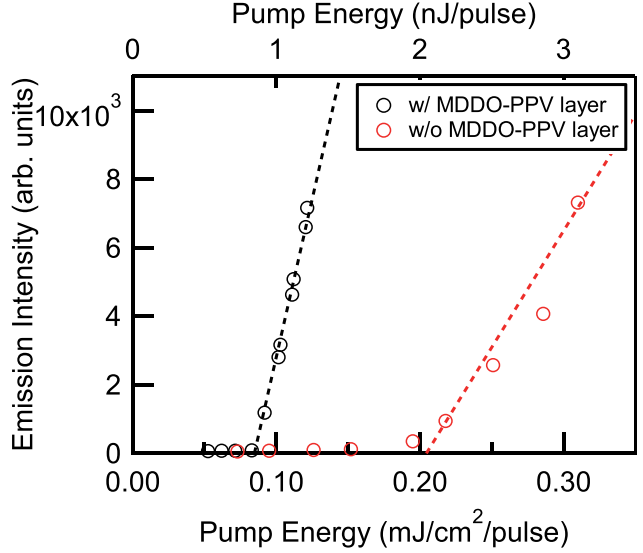

FIG. 5. Laser threshold characteristics of the two ChLC lasers obtained experimentally.

clearly indicates that the sharp emission peak is due to distributed feedback lasing from the helical structure of the $\mathrm{ChLC}{ }^{29}$

Figure 5 shows the threshold characteristics of the ChLC laser. It is clear that the proposed device with the auxiliary active layer has a much lower threshold than the conventional device without the auxiliary active layer. The thresholds were 0.083 and $0.195 \mathrm{~mJ} / \mathrm{cm}^{2} /$ pulse for the device with and without the MDDO-PPV layer, respectively, indicating an improvement in the lasing threshold by a factor of 2.3. The threshold values cannot be compared directly with those reported by other groups, since experimental conditions such as the pulse width, repetition rate, and spot-size of the of the pump beam are different. Nevertheless, to the best of our knowledge, the energy required per pulse $(0.83 \mathrm{~nJ} / \mathrm{pulse})$ is the lowest achieved to date for ChLC lasers, lower by an order of magnitude or more compared to values achieved by other methods, such as band-edge excitation ${ }^{30}$ or doping of high-performance dyes. ${ }^{31}$ This undoubtedly shows the effectiveness of our approach.

We discuss the improvement achieved in the lasing threshold by showing how the photonic DOS and gain of the cavity are modulated by the auxiliary active layer. The DOS determines the threshold gain required for lasing to occur, as it is related to the effective reflectance $R$ at the lasing wavelength

$$
R^{2}=\frac{D O S /(n / c)-1}{D O S /(n / c)+1}
$$

which is further related to the threshold condition for lasing

$$
\alpha_{t h}=\alpha_{l}-\frac{1}{2 L} \ln R^{2},
$$

where $\alpha_{t h}$ is the threshold gain, $\alpha_{l}$ is the loss (which in our calculations has been assumed to be negligible), and $L$ is the cavity length. ${ }^{9}$

Unlike the planar (bulk) ChLC structure in which the DOS can be obtained analytically, it is not so straightforward to obtain the DOS in the ULH structure, because of waveguiding effects. We can, however, obtain the DOS numerically by observing the photon lifetime $\tau_{c}$ in the cold cavity, using the relationship $D O S=\tau_{c} / L$. Figure 6 shows the

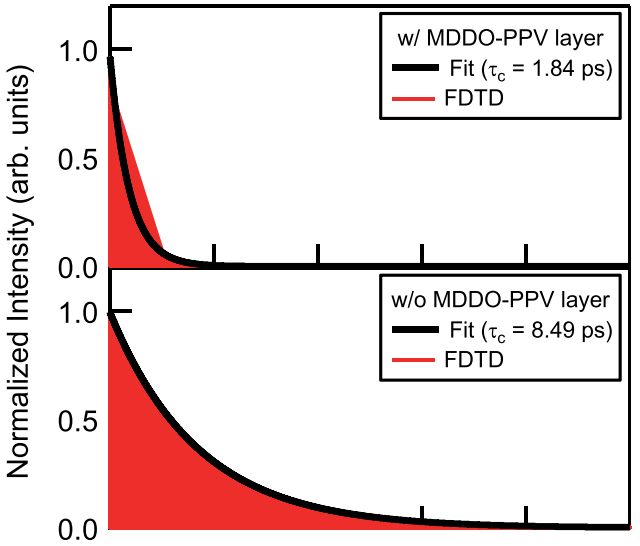

FIG. 6. Light decay from cold cavity (a) with and (b) without the MDDOPPV layer.

results of light decay in the two types of cold cavity and values for $\tau_{c}$, DOS, $R$, and $\alpha_{t h}$ retrieved from the FDTD results are summarized in Table III. It is found that, owing to the presence of the extra high-index layer that cannot contribute to distributed feedback, the DOS is lowered, resulting in an increase in the laser threshold by a factor of 4.66.

We next turn to the gain $\alpha$ achieved in the cavity, and show that while the DOS decreases in the proposed structure, there is enough gain enhancement to result in a net improvement of the threshold. Assuming negligible absorption, the gain coefficient is proportional to the inversion population density

$$
\alpha(x, \omega)=\Delta N(x) \sigma(\omega)
$$

where $\sigma(\omega)$ is the stimulated emission cross section. Since $\sigma(\omega)$ is similar in DCM and MDDO-PPV (see Table II), we can assume the gain to be determined primarily by the inversion population density $\Delta N(x)$ achieved in the cavity.

In Fig. 7, we compare the inversion population density achieved in the devices with and without the MDDO-PPV layer (results shown for two cavities at $t=30 \mathrm{ps}$, pumped at a rate of $P_{r 0}=10 \times 10^{6} \mathrm{~s}^{-1}$ ). In both cavities, the inversion population density decreases exponentially in the depth direction of the cell, reflecting the effect of light absorption. However, in the cavity with MDDO-PPV, the inversion population density increases dramatically, because of the high electron density. The total inversion population densities $\left(\int \Delta N d x / \int d x\right)$ achieved in the devices with and without the MDDO-PPV layer were and $3.00 \times 10^{18} \mathrm{~cm}^{-3}$ and $3.20 \times 10^{17} \mathrm{~cm}^{-3}$, respectively, showing a 9.37 -fold increase in gain with the addition of the gain layer. The net improvement in the lasing threshold is therefore estimated to be

TABLE III. Photon lifetime $\tau_{c}$, DOS, reflectance $R$, and threshold gain coefficient $\alpha_{t h}$ of the devices with and without MDDO-PPV layer.

\begin{tabular}{lcccc}
\hline \hline & $\tau_{c}(\mathrm{ps})$ & $D O S /(n / c)$ & $R$ & $\alpha_{t h}\left(\mathrm{~cm}^{-1}\right)$ \\
\hline MDDO-PPV & 1.84 & 11.5 & 0.916 & 58.2 \\
w/o MDDO-PPV & 8.49 & 53.0 & 0.981 & 12.6 \\
\hline \hline
\end{tabular}




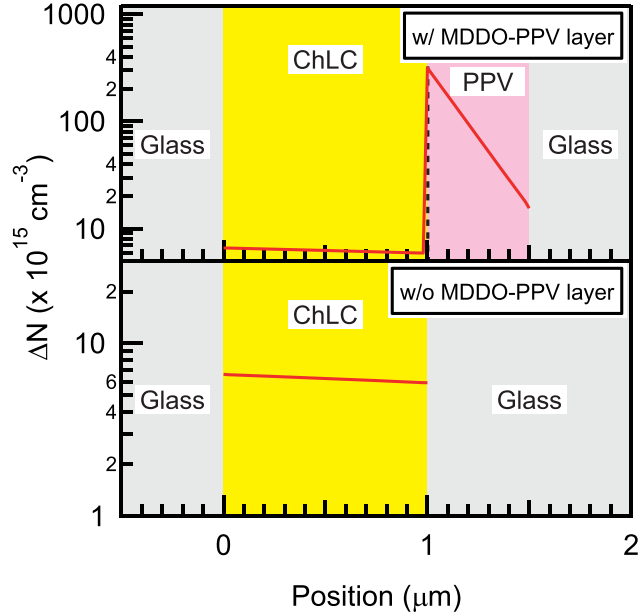

FIG. 7. Inversion population density at pumping rate $P_{r 0}=10 \times 10^{6} \mathrm{~s}^{-1}$ and time $t=30 \mathrm{ps}$ (a) with and (b) without the MDDO-PPV layer.

$9.37 / 4.66=2$-fold, which is in close agreement with the improvement obtained experimentally, 2.3.

\section{v. CONCLUSIONS}

We presented a novel device structure that contains an auxiliary gain layer to improve the lasing threshold of ULH ChLC lasers. We showed through ADE-FDTD simulation that the net improvement in the threshold is achieved as a result of the additional gain, provided from the extra layer, which overcomes the degradation of the cavity quality caused by its addition. Our analyses point to features that are important in further improving the threshold in this device structure: the auxiliary gain layer should be as thin as possible and have a lower index than that of the ChLC to minimize the decrease in the DOS, and should have a high absorption coefficient so that it can provide gain efficiently. For an arbitrary gain medium with particular absorption and emission properties, the optimum thickness for each layer can be designed on the basis of the results of FDTD analysis.

Another noteworthy point to mention is that the proposed device can be pumped electrically, since the gain medium is located outside the LC, which is an insulator. While systems with a DFB cavity located on top of a $\pi$-conjugated polymer are commonly studied in the field of organic lasers, ${ }^{32}$ the ULH ChLC cavity is advantageous over other types of cavity since the periodicity can be tuned continuously by applying an electric field. ${ }^{33}$ The development of systems in which the pump and feedback can be controlled independently is crucial for the realization of electrically pumped, tunable organic lasers. We believe that the device structure we propose is a step towards achieving this goal.

\section{ACKNOWLEDGMENTS}

This work was supported by JSPS KAKENHI Grant No. A228590. H. Yoshida acknowledges support from the JST PRESTO Program.

${ }^{1}$ I. P. Il'chishin, E. A. Tkhonov, V. G. Tishchenko, and M. T. Shpak, JETP Lett. 32, 27 (1980).

${ }^{2}$ H. J. Coles and S. M. Morris, Nat. Photonics 4, 676 (2010).

${ }^{3}$ Y. Inoue, H. Yoshida, K. Inoue, Y. Shiozaki, H. Kubo, A. Fujii, and M. Ozaki, Adv. Mater. 23, 5498 (2011).

${ }^{4}$ J. Schmidtke, W. Stille, and H. Finkelmann, Phys. Rev. Lett. 90, 083902 (2003).

${ }^{5}$ M. H. Song, N. Y. Ha, K. Amemiya, B. Park, Y. Takanishi, K. Ishikawa, J. W. Wu, S. Nishimura, T. Toyooka, and H. Takezoe, Adv. Mater. 18, 193 (2006).

${ }^{6}$ H. Yoshida, C. Lee, Y. Matsuhisa, A. Fujii, and M. Ozaki, Adv. Mater. 19, 1187 (2007).

${ }^{7}$ Y. Matsuhisa, R. Ozaki, M. Ozaki, and K. Yoshino, Jpn. J. Appl. Phys., Part 2 44, L629 (2005).

${ }^{8}$ Y. Matsuhisa, R. Ozaki, K. Yoshino, and M. Ozaki, Appl. Phys. Lett. 89, 101109 (2006).

${ }^{9}$ L. M. Blinov, JETP Lett. 90, 166 (2009).

${ }^{10}$ W. Cao, P. Palffy-Muhoray, B. Taheri, A. Marino, and G. Abbate, Mol. Cryst. Liq. Cryst. 429, 101 (2005)

${ }^{11}$ H. Yoshida, Y. Inoue, T. Isomura, Y. Matsuhisa, A. Fujii, and M. Ozaki, Appl. Phys. Lett. 94, 093306 (2009).

${ }^{12}$ Y. Inoue, H. Yoshida, K. Inoue, A. Fujii, and M. Ozaki, Appl. Phys. Express 3, 102702 (2010).

${ }^{13}$ M. Uchimura, Y. Watanabe, F. Araoka, J. Watanabe, H. Takezoe, and G. Konishi, Adv. Mater. 22, 4473 (2010).

${ }^{14}$ A. Taflove and S. C. Hagness, Computational Electrodynamics: The Finite-Difference Time-Domain Method, 3rd ed. (Artech House, Norwood, MA, 2005).

${ }^{15}$ A. E. Siegman, Lasers (University Science Books, Sausalito, CA, 1986), Chaps. 2, 3, and 6 .

${ }^{16}$ A. S. Nagra and R. A. York, IEEE Trans. Antennas Propag. 46, 334 (1998).

${ }^{17}$ T. Matsui and M. Kitaguchi, Appl. Phys. Express 3, 061701 (2010).

${ }^{18}$ K. Funamoto, M. Ozaki, and K. Yoshino, Jpn. J. Appl. Phys., Part 242 , L1523 (2003).

${ }^{19}$ M. Hamaguchi and K. Yoshino, Jpn. J. Appl. Phys., Part 2 33, L1478 (1994).

${ }^{20}$ M. Hamaguchi and K. Yoshino, Jpn. J. Appl. Phys., Part 2 34, L712 (1995).

${ }^{21}$ K. S. Yee, IEEE Trans. Antennas Propag. 14, 302 (1966).

${ }^{22}$ X. Jiang and C. Soukoulis, Phys. Rev. Lett. 85, 70 (2000).

${ }^{23}$ A. Yariv and P. Yeh, Photonics: Optical Electronics in Modern Communications, 6th ed., The Oxford Series in Electrical and Computer Engineering (Oxford University Press, New York, 2007).

${ }^{24}$ R. Kersting, U. Lemmer, R. Mahrt, K. Leo, H. Kurz, H. Bäaessler, and E. Göbel, Phys. Rev. Lett. 70, 3820 (1993).

${ }^{25}$ M. Watanabe, N. Yamasaki, T. Nakao, K. Masuyama, H. Kubo, A. Fujii, and M. Ozaki, Synth. Met. 159, 935 (2009).

${ }^{26} \mathrm{O}$. Svelto and D. C. Hanna, Principles of Lasers, 5th ed. (Springer, New York, 2009).

${ }^{27}$ C. Mowatt, S. M. Morris, M. H. Song, T. D. Wilkinson, R. H. Friend, and H. J. Coles, J. Appl. Phys. 107, 043101 (2010).

${ }^{28}$ J. P. Berenger, J. Comput. Phys. 114, 185 (1994).

${ }^{29}$ V. I. Kopp, B. Fan, H. K. M. Vithana, and A. Z. Genack, Opt. Lett. 23, 1707 (1998).

${ }^{30}$ Y. Matsuhisa, Y. Huang, Y. Zhou, S.-T. Wu, R. Ozaki, Y. Takao, A. Fujii, and M. Ozaki, Appl. Phys. Lett. 90, 091114 (2007).

${ }^{31}$ F. Araoka, K.-C. Shin, Y. Takanishi, K. Ishikawa, H. Takezoe, Z. Zhu, and T. M. Swager, J. Appl. Phys. 94, 279 (2003).

${ }^{32}$ S. Chénais and S. Forget, Polym. Int. 61, 390 (2012).

${ }^{33}$ Y. Inoue, Y. Matsuhisa, H. Yoshida, R. Ozaki, H. Moritake, A. Fujii, and M. Ozaki, Mol. Cryst. Liq. Cryst. 516, 182 (2010). 